\title{
Cuidados de enfermagem para prevenção de infecção do trato urinário em pacientes com cateterismo vesical de demora (CVD) no ambiente hospitalar
}

\author{
Nursing care for prevention of urinary tract infection in patients with bladder catheterization \\ in the hospital environment
}

\author{
Jamille Santos de Jesus ${ }^{1}$, Mônica Franco Coelho ${ }^{2}$, Reginaldo Adalberto Luz ${ }^{3}$
}

\section{Resumo}

Objetivo: Identificar na literatura os cuidados de enfermagem para prevenção de infecção do trato urinário em pacientes com cateterismo vesical de demora, no ambiente hospitalar. Método: Estudo bibliográfico descritivo de abordagem quantitativa. Realizado em maio de 2016, no site da Biblioteca Virtual em Saúde, nas bases de dados Scientific Eletronic Library Online e Literatura Latino-Americano em Ciências de Saúde. Resultados: Dos 04 artigos utilizados, todos $(100 \%)$ foram elaborados por profissionais da área da enfermagem, na categoria de enfermeiro. Os cuidados de enfermagem para a prevenção de ITU foram divididos em duas temáticas. Na primeira temática estão os cuidados de enfermagem relacionados a técnica do CVD. Na segunda temática estão os cuidados de enfermagem na manutenção do CVD. Conclusão: No presente estudo evidenciou-se que o enfermeiro é o principal responsável para conscientizar a equipe sobre os cuidados para minimizar os riscos de ITU associada ao CVD. Sendo o enfermeiro a chave para a avaliação da pertinência do uso continuado do cateter, identificando as complicações e implementação de práticas de cuidados para minimizar as complicações.

Descritores: Cateterismo urinário, cuidados de enfermagem

\section{Abstract}

Objective: Identify in the literature the nursing care for

1. Acadêmica da Faculdade de Ciências Médicas da Santa Casa de São Paulo. $8^{\circ}$ Semestre do Curso de Graduação em Enfermagem

2. Professora da Universidade Paulista

3. Professor Instrutor da Faculdade de Ciências Médicas da Santa Casa de São Paulo. Curso de Graduação em Enfermagem Trabalho realizado: Faculdade de Ciências Médicas da Santa Casa de São Paulo - Curso de Graduação em Enfermagem

Endereço para correspondência: Jamille Santos de Jesus. Rua Dr. Cesário Mota Jr, 61, $9^{\circ}$ andar - Vila Buarque - 01221-020 - São Paulo-SP - Brasil.E-mail: jamillysantos89@hotmail.com prevention of urinary tract infection in patients with catheterization and bladder, in the hospital environment. Method: Descriptive bibliographic study of quantitative approach. Held in May 2016, on the site of the Virtual Health Library, in the databases Scientific Eletronic Library Online and Latin American Literature in Health Sciences. Results: Of the 04 articles used, all (100\%) were elaborated by professionals of the nursing area, in the category of nurse. Nursing care for the prevention of UTIs was divided into two thematics. In the first thematic are the nursing care related to the $B C D$ technique. The second thematic is nursing care in the maintenance of $B C D$. Conclusions: In the present study it was evidenced that the nurse is the main responsible for making the team aware of the care to minimize the risks of UTI associated with BCD. Being the nurse the key to the evaluation of the pertinence of the continued use of the catheter, identifying the complications and the implementation of care practices to minimize the complications.

Keywords: Urinary catheterization and nursing care

\section{Introdução}

A infecção relacionada a assistência à saúde (IRAS) é qualquer processo infeccioso adquirido pelo paciente após sua admissão nos serviços de saúde, podendo se manifestar durante a internação ou após a alta, desde que esteja associada a internação ou procedimentos. Também são consideradas IRAS aquelas infecções que se manifestam antes de 72 horas de internação e podem ser relacionadas com a realização de procedimentos diagnósticos e/ou terapêuticos, realizado durante este período ${ }^{(1)}$.

Estudo realizado nos Estados Unidos identificou as infecções mais frequentes em uma unidade de terapia intensiva, a primeira foi a pneumonia, seguida por infecção do trato urinário (ITU) e infecção de corrente sanguínea, estas últimas relacionadas diretamente a manipulação de cateter vesical e de punção periférica respectivamente ${ }^{(2)}$. 
A ITU é caracterizada por um quadro infeccioso que acomete qualquer parte do sistema urinário como: rins, ureteres, uretra e bexiga ${ }^{(3)}$.

O cateterismo vesical é um procedimento invasivo, no qual se introduz um cateter vesical pelo canal uretral até a bexiga do paciente com a finalidade da drenagem da urina. Pode ser classificado como cateterismo vesical de alívio, intermitente e de demora ${ }^{(4)}$.

No Brasil a ITU é responsável por cerca de 35 a $45 \%$ de todas as infecções adquiridas no ambiente hospitalar, sendo que $80 \%$ estão associadas ao uso do $\mathrm{CVD}^{(5)}$. E caracteriza-se como a segunda infecção mais frequente na população geral, independente da faixa etária ${ }^{(6)}$.

Dados epidemiológicos destacam que em média $10 \%$ dos pacientes que foram cateterizados apresentaram bacteriúria no momento do procedimento e dos pacientes que não apresentaram o problema cerca de $10 \%$ a $20 \%$ desenvolverão bacteriúria durante a permanência com o cateter ${ }^{(6)}$.

Através de estudos científicos, muitas modificações se fizeram necessárias na prática da assistência aos pacientes com cateter urinário, para diminuir as taxas de ITU. Medidas especialmente dirigida a técnica de inserção, ao sistema coletor, ao tipo de cateter, aos cuidados diários $^{(7)}$.

OCVD é considerado um procedimento complexo e que necessita da aplicação de conhecimentos científicos ao executar. Conforme a Resolução n ${ }^{\circ} 450 / 2013$, publicada em dezembro pelo Conselho Federal de Enfermagem (COFEN), a inserção do cateter vesical é função privativa do enfermeiro ${ }^{(8)}$.

A prevenção diminui as taxas de morbidade, mortalidade e custos no tratamento da ITU associada ao uso de CVD. Em face do contexto apresentado o presente estudo tem como objetivo identificar os cuidados de enfermagem para prevenção de ITU em pacientes submetidos ao cateterismo vesical no ambiente hospitalar.

\section{Objetivo}

Identificar na literatura os cuidados de enfermagem para prevenção de infecção do trato urinário em pacientes com cateterismo vesical, no ambiente hospitalar.

\section{Método}

Trata-se de um estudo bibliográfico descritivo de abordagem quantitativa. Foi desenvolvido com base em material já elaborado, constituído principalmente de livros, artigos científicos e tese e nas bases de dados Biblioteca Virtual em Saúde (BVS)- Literatura Latino-Americano em Ciências de Saúde (LILACS) e no site da Scientific Eletronic Library Online- SciELO.
A indexação do material referente à busca foi realizada, utilizando os descritores: cateterismo urinário e cuidados de enfermagem.

Através das ferramentas de filtro disponíveis nos portais de busca, limitados aos artigos escritos em português cuja afiliação dos autores seja de instituições no Brasil. Trabalhos científicos publicados entre os anos de 2005 a 2015, escritos em português, disponível gratuitamente e na íntegra.

\section{Resultados}

Foram obtidos inicialmente, com base nos critérios de inclusão e exclusão já descritos, 19 artigos. Após a leitura dos títulos e resumos, foram selecionados 16 artigos para leitura da íntegra para avaliar o conteúdo de cada artigo a ser inserido neste trabalho. A amostra final da revisão foi composta por quatro artigos e uma tese. Para facilitar a apresentação e organização dos resultados foram estabelecidas duas temáticas como descritas a seguir:

\section{1 - Principais medidas relacionadas à técnica do cateterismo vesical para prevenção de ITU}

Obteve-se nesta categoria o total de quatro $(80 \%)$ trabalhos que detalham aspectos específicos da técnica de cateterismo vesical desde a lavagem das mãos antes do procedimento até a fixação do cateter ${ }^{(9-11)}$.

Três trabalhos citam a importância do uso de luvas estéreis ${ }^{(10,12)}$ e três trabalhos citam a técnica asséptica como medidas para minimizar o risco de ITU durante o procedimento de $\mathrm{CVD}^{(11,13)}$. O uso de gel lubrificante apareceu em dois estudos como mediadas preventivas para ITU ${ }^{(11,12)}$. A utilização do campo fenestrado apareceu em dois estudos como medidas para minimizar os riscos de ITU relacionados a inserção do $\mathrm{CVD}^{(10-11)}$. Apenas um $(20 \%)$ trabalho trata sobre a importância da avaliação diária do paciente para identificar se há necessidade da sondagem e a manutenção do CVD o menor tempo possível ${ }^{(10)}$.

Apenas 02 (40\%) estudos citaram a higienização das mãos como cuidados de enfermagem para diminuição do risco de ITU ${ }^{(11-12)}$.

\section{2 - Cuidados de enfermagem na manutenção do cateter vesical}

Em quatro (80\%) dos trabalhos, a fixação adequada aparece como uma medida importante para a manutenção do $\mathrm{CVD}^{(9,11)}$. A manutenção do fluxo urinário através da manutenção do CVD desobstruído foi apresentada em dois $(40 \%)$ dos trabalhos ${ }^{(9,13)}$. A realização da higiene íntima do paciente pelo menos uma vez ao dia apareceu em dois (40\%) trabalhos ${ }^{(10-11)}$. A manutenção do sistema de drenagem fechado um (20\%) trabalho. 
A maioria dos trabalhos, quatro $(80 \%)$ determinaram a importância de se manter a bolsa coletora abaixo do nível da bexiga ${ }^{(9,11)}$. Três $(60 \%)$ estudos enfatizaram que a bolsa coletora deve ser mantida de forma suspensa na cama sem ter contato com o chão e outros objetos ${ }^{(11,13)}$

\section{Discussão}

Observou-se em todos os trabalhos científicos que os autores principais eram enfermeiros. Ressalta-se ainda a importância do enfermeiro e sua participação ativa no controle e na elaboração de rotinas com vistas a redução das infecções relacionadas a assistência à saúde, dentre as quais estão inseridas as infecções de trato urinário ${ }^{(14)}$.

Nos trabalhos científicos levantados foi possível identificar dois aspectos prevalentes, o rigor na técnica asséptica durante a realização do procedimento de CVD e os cuidados com a manutenção do dispositivo que foram elencados em duas categorias temáticas distintas. Estes aspectos foram categorizados como: principais medidas relacionadas a técnica do cateterismo vesical para prevenção de ITU e cuidados de enfermagem na manutenção do cateter vesical, respectivamente e que serão discutidos a seguir.

\section{Principais medidas relacionadas à técnica do cateterismo vesical para prevenção de ITU.}

Embora o CVD seja realizado pelos enfermeiros verifica-se na prática que esta técnica apresenta condutas não padronizadas, sendo realizadas de maneiras distintas entre as instituições de saúde ${ }^{(4)}$.

A realização do CVD em um ambiente tranquilo e que garanta a não exposição do paciente, durante o procedimento, evitando exposição desnecessária e zelando pelo conforto é uma atribuição do enfermeiro ${ }^{(15)}$.

No que se refere a higienização das mãos esta etapa, muito importante para a prevenção de ITU, não foi considerada em todos os trabalhos. A higienização das mãos deve ser realizada antes e após a inserção do cateter ou manipulação do períneo para remoção dos microrganismos transitórios que residem na camada mais superficial da epiderme ${ }^{(15)}$. No presente estudo a higiene íntima antes do cateterismo urinário foi citada por dois autores como medidas importantes para diminuição da ITU associada a cateterização vesical ${ }^{(10-11)}$.

Quanto à realização da antissepsia, tal procedimento foi descrito como importante para a prevenção da ITU relacionada a inserção do cateter em apenas um $\operatorname{artigo}^{(11)}$.

Quanto a utilização de campo fenestrado estéril durante o procedimento, foi considerado relevante em dois trabalhos ${ }^{(10-11)}$.
A utilização de gel lubrificante foi citada em menos da metade dos trabalhos ${ }^{(11-12)}$.

Outros aspectos que previnem a lesão da mucosa uretral e ITU associada ao procedimento são a escolha adequada do calibre do cateter e a garantia da insuflação do balonete com segurança após a visualização da drenagem de urina logo após a inserção do dispositi$\mathrm{vo}^{(4)}$. Tais aspectos não foram abordados em nenhum dos trabalhos identificados neste estudo.

Dos trabalhos levantados três foram publicados após o ano de 2013, destes a maioria, dois, trataram da questão do CVD ser uma conduta específica do enfermeiro enquanto um dos trabalhos não mencionou esta questão.

\section{Cuidados de enfermagem na manutenção do cateter vesical.}

A medida mais eficaz que a equipe de saúde pode adotar após a inserção do cateter é reavaliar constantemente a necessidade da permanência do cateter e mantê-lo apenas o tempo necessário e com a definição clara do motivo pelo qual foi solicitada ${ }^{(6)}$.

$\mathrm{O}$ enfermeiro tem uma participação ativa na avaliação para a retirada do CVD. Neste trabalho, apenas um autor referiu a importância da reavaliação diária da necessidade do cateter e identificar sinais e sintomas indicativos de ITU ${ }^{(10)}$.

A equipe de enfermagem deve estar atenta quanto ao posicionamento da bolsa coletora, isto foi identificado na maioria dos trabalhos deste estudo que ressaltaram a necessidade de se manter a bolsa coletora de urina posicionada abaixo do nível da bexiga do paciente e sem encostar-se a outros objetos ou no chão ${ }^{(9,11)}$. Esta informação corresponde a resultado de outro estudo que avaliou os fatores predisponentes para ocorrência de ITU em pacientes críticos no qual um dos critérios para a boa manutenção do CVD foi a observação do posicionamento da bolsa coletora abaixo do nível da bexiga ${ }^{(5)}$. A higiene íntima durante a permanência do indivíduo com o CVD é uma medida efetiva na prevenção da ITU, nos trabalhos que citaram este aspecto relacionado ao cuidado com o dispositivo a recomendação foi de uma vez ao dia ${ }^{(10,11)}$. Em relação ao detalhamento desta técni$\mathrm{ca}$, apenas um dos autores indica o uso de sabão e água para realização da higiene íntima ${ }^{(10)}$.

Segundo Mazzo (2014)(4) a higienização do meato uretral deve ser realizada com água e sabão pelo menos uma vez ao dia, durante a realização do banho. Segundo estudo a higiene do períneo tem se mostrado como medida eficaz para a diminuição da incidência de ITU ${ }^{(4)}$

Após a inserção de um cateter vesical de demora, deve-se manter um sistema de drenagem urinária fechado para minimizar os riscos de infecção. Uma possível interrupção do sistema leva à introdução de microrga- 
nismo. É recomendado que a bolsa de drenagem seja esvaziada pelo menos a cada 8 horas e caso perceba grandes eliminações esse intervalo deve ser reduzido, assim como deve ser assegurado que cada paciente tenha um recipiente separado para a medição de urina, prevenindo a contaminação cruzada ${ }^{(16)}$.

\section{Conclusão}

Este estudo permitiu identificar os cuidados de enfermagem para prevenção de infecção do trato urinário em pacientes com CVD, categorizadas em: medidas relacionadas à técnica do cateterismo vesical para prevenção de ITU e cuidados de enfermagem na manutenção do cateter vesical.

A inserção do cateter urinário é um dos principais problemas relacionados à infecção hospitalar da atualidade. Sendo de fundamental importância a participação de todos da equipe de saúde, para adoção de medidas preventivas.

No presente estudo evidenciou-se que o enfermeiro é o principal responsável para conscientizar a equipe sobre os cuidados para minimizarem os riscos de ITU associada ao CVD. Sendo o enfermeiro a chave para a avaliação da pertinência do uso continuado do cateter, identificando as complicações e implementação de práticas de cuidados para minimizar as complicações.

Em relação às ações de enfermagem, pode-se identificar no conjunto dos trabalhos os possíveis atos preventivos de infecção associada ao cateterismo vesical destacado no estudo. Medidas simples como a lavagem das mãos, uso de técnica asséptica, uso de lubrificantes, manutenção do sistema fechado e posicionamento da bolsa de drenagem são algumas das medidas que minimizam os riscos de ITU.

\section{Referências}

1. Sousa CMM, Alves MSCF, Moura MEB, Silva AO. Os direitos dos usuários da saúde em casos de infecção hospitalar. Rev Bras Enferm. 2008; 61(4):411-7.

2. Moura MEB, Campelo SMA, Brito FCP, Batista OMA, Araújo TME, Oliveira ADS. Infecção hospitalar: estudo de prevalência em um hospital público de ensino. Rev Bras Enferm. 2007 ; 60(4):416-21.

3. Smeltzer SC, Bare BG, Hinkle JL, Cheever KH. Cuidados aos pacientes com distúrbios urinários. In: Smeltzer SC, Bare BG, Hinkle JL, Cheever KH, editors. Brunner \& Suddarth: tratado de enfermagem médico-cirúrgica. $11^{\mathrm{a}}$ ed. Rio de Janeiro: Guanabara Koogan, 2009. v.3, p.1327-59.
4. Mazzo A, Coelho MF, Jorge BM, Cassini M, Mendes IAC, Martins JCA, et al. Enfermagem na abordagem das infecções geniturinárias. In: Associação Brasileira de Enfermagem; Bresciani HR, Martini JG, Mai LD, organizadores. PROENF Programa de Atualização em Enfermagem: Saúde do Adulto: Ciclo 9. Porto Alegre: Artmed/Panamericana; 2014. p. 29-47.

5. Menegueti MG, Martins MA, Canini SEM, Basile Filho A, Laus AM. Infecção urinária em unidade de terapia intensiva: um indicador de processo para a prevenção. Rev Rene. 2012; 13(3):632-8.

6. Queiros MI, Cipriano MAB, Santos MCL, Cardozo MVLML. Infecções urinárias e uso de cateter vesical de demora em unidade pediátrica. Rev Rene. 2011;12(2):295-301.

7. Conterno LO, Lobo JA, Masson W. Uso excessivo do cateter vesical em pacientes internados em enfermarias de hospital universitário. Rev Esc Enferm USP. 2011; 45(5):1089-96.

8. Conselho Federal de Enfermagem. Decreto $n^{\circ}$ 0450/2013. Normatiza o procedimento de Sondagem Vesical no âmbito do Sistema COFEN / Conselhos Regionais de Enfermagem. [online]. Brasília: COFEN; 2013. Disponível em: http://www. cofen.gov.br/resolucao-cofen-no-04502013-4_23266.html [03 ago 2016]

9. Moraes CL, Chaves NMO. Controle de infecção em cateterismo vesical de demora em unidade de terapia intensiva. Rev Enf Cent O Min. 2015;5(2):1650-7.

10. Campos CC. Incidência de infecção do trato urinário relacionada ao uso do cateter urinário de demora em centros de terapia intensiva: comparação entre duas técnicas de limpeza periuretral. Dissertação [Mestrado]. Belo Horizonte: Universidade Federal de Minas Gerais. Escola de Enfermagem; 2014.

11. Souza ACS, Tipple AF, Barbosa JM, Pereira MS, Barreto RASS. Cateterismo urinário: conhecimento e adesão ao controle de infecção pelos profissionais de enfermagem. Rev Eletronica Enf. 2007; 9(3):724-35.

12. Torres GV, Fonseca PCB, Costa IKF. Cateterismo vesical de demora como fator de risco para infecção do trato urinário: conhecimento da equipe de enfermagem de unidade de terapia intensiva. Rev Enferm UFPE Online. 2010; 4(2):453-60.

13. Shimabukuru PMS, Paulon P, Feldman LB. Implantação de bundles em unidade de terapia intensiva: um relato de experiência. Rev Enferm UFSM. 2014; 4(1):227-36.

14. Brasil. Ministério da Saúde. Portaria Federal/GM/MS n ${ }^{\circ}$ 2.616/1998 de 12 de maio de 1998. Dispõe diretrizes e normas para a prevenção e o controle infecções hospitalares. Brasília: Ministério da Saúde; 1998.

15. Centers for Disease Control and Prevention. Guideline for Hand Hygiene in Health-Care Settings: Recommendations of the Healthcare Infection Control Practices Advisory Committee and the HICPAC/SHEA/APIC/IDSA Hand Hygiene Task Force. MMWR Recomend Reprod. 2002; 51(RR-16):1-51.

16. Potter PA, Perry AG. Eliminação urinária. In: Potter PA, Perry AG. Fundamentos de enfermagem. Rio de Janeiro: Elsevier; 2009. p. 1129-73.

Trabalho recebido: 01/03/2017

Trabalho aprovado: 17/05/2018 